\title{
BMJ Open Methamphetamine and cannabis abuse in adolescence: a quasi-experimental study on specific and long-term neurocognitive effects
}

\author{
Natalie L Cuzen, ${ }^{1}$ Sheri-Michelle Koopowitz, ${ }^{1}$ Helen L Ferrett, ${ }^{2}$ Dan J Stein, ${ }^{1}$ \\ Deborah Yurgelun-Todd ${ }^{3,4}$
}

To cite: Cuzen NL Koopowitz S-M, Ferrett HL, et al. Methamphetamine and cannabis abuse in adolescence: a quasiexperimental study on specific and long-term neurocognitive effects. BMJ Open 2015;5:e005833. doi:10.1136/bmjopen-2014005833

- Prepublication history for this paper is available online. To view these files please visit the journal online (http://dx.doi.org/10.1136/ bmjopen-2014-005833).

Received 20 June 2014 Revised 28 September 2014 Accepted 6 November 2014

CrossMark

For numbered affiliations see end of article.

Correspondence to

Dan J Stein;

dan.stein@uct.ac.za

\section{ABSTRACT}

Objectives: Methamphetamine abuse affects brain structure and function. Although methamphetamine and cannabis are commonly abused together, few studies have investigated the differential neurocognitive consequences of methamphetamine abuse with or without cannabis. Furthermore, the effects of drug use on the developing adolescent brain remain poorly understood. We compared neurocognitive function between adolescents with 'pure' methamphetamine abuse, those with comorbid methamphetamine and cannabis abuse, and healthy controls at baseline and follow-up.

Methods: Individuals residing in the greater Cape Town region, between the ages of 13 and 18 years, were recruited into either Methamphetamine only group (Meth-only; $n=10$ ), Methamphetamine and cannabis group (Meth-cann; $n=10$ ) or healthy control $(n=20)$ groups using a quasi-experimental design. All participants underwent a comprehensive neurocognitive assessment. Substance-use variables and psychiatric symptom counts were also recorded. A portion of the Meth-only and control participants completed 12-month follow-up assessments.

Results: While the Meth-cann group demonstrated widespread neurocognitive deficits at baseline, these deficits were restricted to the self-monitoring domain in the Meth-only group at baseline and at follow-up.

Conclusions: Methamphetamine abuse with cannabis abuse is associated with significantly more neurocognitive impairment than methamphetamine abuse alone, and such deficits may be enduring.

\section{INTRODUCTION}

Preclinical and human data suggest that various brain regions and neurocognitive functions may be differentially vulnerable to the effects of regular heavy methamphetamine use. ${ }^{1-4}$ For instance, human studies have shown that methamphetamine abuse may lead to broad and long-term neurocognitive sequelae in memory, attention, task

\section{Strengths and limitations of this study}

- Identification of neurocognitive sequelae in the early phase of drug abuse.

- Identification of neurocognitive sequelae of relatively 'pure' drug abuse.

- Findings specific to adolescent phase.

- Limited sample size of all three groups.

switching and response inhibition domains, and that such alternations in brain function may persist for years following abstinence. . $^{5-7}$ Furthermore, the offspring of methamphetamine abusing mothers suffer lasting damaging effects on cognitive development. ${ }^{8-10}$ There are a number of reviews that explore the neurocognitive effects of drug use (particularly methamphetamine or cannabis) in adolescents. ${ }^{11-13}$ Marijuana-using adolescents have displayed neurocognitive deficits in the memory and executive functioning domains. ${ }^{13}$ Furthermore, in a recent study, it was found that the primary deficit among methamphetamine-using adolescents is executive functioning. ${ }^{14}$ However, these studies and many reviews focus on single drug-using adolescents. Many studies do not explore the combination of cannabis and methamphetamine use in adolescents, and the neurocognitive effects this combination has on the developing brain.

In addition to overall severe and persistent neurocognitive effects of heavy methamphetamine use, the adolescent brain may be particularly vulnerable to such effects given critical neuromaturation underway during this phase of development. ${ }^{15}{ }^{16}$ However, few data specifically address the question of neurocognitive sequelae of methamphetamine abuse in adolescents during the early stage of drug use trajectory. Over the 1990s and into the present, substance abuse statistics 
have revealed alarming increases in methamphetamine abuse rates to the extent that methamphetamine abuse may be considered an 'epidemic' in the USA. ${ }^{17}$ Increases in methamphetamine abuse have been noted elsewhere as well: methamphetamine has recently attained the status of primary drug of abuse among adolescents in developing countries such as South Africa. ${ }^{18}$ This represents a dramatic shift in drug use patterns in this age group in recent years, and raises issues about the impact of methamphetamine on the developing brain during this critical time of brain development.

Despite overall increased rates of methamphetamine abuse, several fundamental questions about the consequences of such abuse remain incompletely answered, thus calling for more empirical work in the area. First, although drug coadministration is a common trend in worldwide patterns of drug abuse, ${ }^{18}$ the differential consequences of methamphetamine abuse with or without cannabis use on adolescent brain function and structure are not yet clearly delineated. Second, while externalising psychopathology commonly co-occurs with heavy methamphetamine use, the functional impact of such comorbidity remains unclear. ${ }^{19}{ }^{20}$ Lastly, the long-term neurocognitive outcome of adolescent methamphetamine abuse with minimal externalising psychopathology remains an open question.

Given the worldwide pattern of drug coadministration, it is particularly challenging to examine the impact of single or specific drug use combinations in most settings. However, in the Cape Town region of South Africa, we are presented with a population of adolescent drug users who name methamphetamine as their sole drug of abuse. In this region, crystal methamphetamine is referred to as 'Tik', an onomatopoeic term in the local lingua franca, Afrikaans, for the sound made by heated crystals in glass receptacles used by the local community to heat and inhale methamphetamine vapours.

Our focus in this report is a neurocognitive comparison between adolescents abusing methamphetamine alone, those abusing methamphetamine and cannabis, and healthy controls with no history of substance misuse. We also examine drug-related changes in these measures over time by presenting follow-up data for a portion of the original sample. We supplement the primary neurocognitive investigation with clinical data on subthreshold (including externalising) psychopathology. We hypothesised that cognitive impairment would be worse in the combined group ('Meth-cann' group) when compared to the other groups.

\section{METHODS}

\section{Participants}

We recruited 13-18-year-old English-speaking and Afrikaans-speaking adolescents of low socioeconomic status and mixed ancestry from the Cape Town region in South Africa. Participants in the initial cohort $(\mathrm{N}=40)$ were recruited into either the (1) ('Meth-only group'; $\mathrm{n}=10)$; (2) Methamphetamine and cannabis group ('Meth-cann group'; $\mathrm{n}=10$ ); or (3) control group $(n=20)$, matched for age, level of education and socioeconomic status. Participants were divided into groups based on information elicited during a clinical interview including administration of the Schedule for Affective Disorders and Schizophrenia for School Aged Children (6-18 years) Lifetime Version (K-SADS-PL ${ }^{21}$ ) and Diagnostic and Statistical Manual of Mental Disorders, Fourth Edition, Text Revision (DSM-IV-TR) ${ }^{22}$ criteria for substance dependence. Participants in the Meth-only group endorsed diagnoses of methamphetamine abuse or dependence with current and regular methamphetamine use (more than three doses per week for the past 6 months) alongside minimal (ie, subdiagnostic threshold and low lifetime consumption) alcohol, cannabis and other substance use; participants in the Meth-cann group endorsed diagnoses of methamphetamine and cannabis abuse or dependence, with current and regular methamphetamine and cannabis use (more than three doses per week for the past 6 months), alongside minimal alcohol and other substance use; the control group comprised mostly non-users (individuals with no exposure to methamphetamine, cannabis or alcohol) and a smaller number of light users (lifetime history of less than 10 doses of any illicit substances), all without past or current history of substance use disorders.

Exclusionary criteria for study participation included first language other than English or Afrikaans; current or past DSM-IV Axis I disorder including major depression, psychosis and anxiety, and eating, conduct, childhood, pervasive developmental and attention deficit/ hyperactivity disorders (ADHD); current or past use of psychotropic medication; alcohol use in excess of 8 units per month for any period of 2 months or more; a positive breathalyser result at assessment visits; evidence or report of significant medical, physical, neurological or infectious conditions that may affect assessments of brain function (eg, diabetes, developmental delays, fetal alcohol spectrum disorders, hepatitis B or C, HIV+); history of head injury with loss of consciousness for over 5 min; history of electroconvulsive therapy treatment; history of placement in special needs classes or academic streaming as a result of poor school performance (estimated IQ<75); left-handedness; poor vision, claustrophobia or metal implant in the body (included for the purposes of the imaging portion of the larger study). Additional exclusionary criteria for participant groups included a negative urinary drug screen result for Meth-only group or Meth-cann group; a positive urinary drug screen result for any illicit substance (control group); a positive urinary drug screen for any other illicit substance aside from methamphetamine or cannabis where relevant (all groups). Aside from these criteria, individuals who had experimented with other drugs were not excluded, provided that they did not 
endorse diagnostic criteria for current or past substance use disorder (SUD), and had not consumed more than 10 doses of the drug in their lifetime or used the drug in the previous 6 months.

A portion of participants in the original control group $(n=10)$ as well as all but one participant in the Meth-only group ( $\mathrm{n}=9)$ were followed-up 12 months after initial recruitment to examine substance use trajectory, as well as changes in neurocognitive measures over time.

\section{Measures}

Psychopathology

To minimise the possible confounding effects of psychopathological comorbidity on neurocognitive measures, our primary outcome measures of interest, we recruited only individuals with relatively modest behavioural problems (ie, not meeting diagnostic criteria for non-substance-related disorders). In order to characterise subtle psychopathology, however, we chose to sum total subdiagnostic threshold symptom counts from the K-SADS-PL for post-traumatic stress disorder, ADHD, conduct disorder (CD) and oppositional-defiant disorder (ODD). Symptom counts for CD and ODD were tallied to form a composite variable representing subdiagnostic externalising pathology (range $=0-20$ ). Previous studies have demonstrated the benefit of adopting similar means of assessing psychopathology in drug-using samples. ${ }^{23-25}$ ADHD symptoms were assessed separately, and were not included in the composite externalising score, based on evidence suggesting that ADHD is inconsistently related to SUD severity, for example, ${ }^{26}$ and that the diagnosis falls outside the classification of disruptive behavioural disorders categorised by social deviance. ${ }^{27}$ In addition, self-reported indices of anxiety were recorded using the Multidimensional Anxiety Scale for Children $\left(\right.$ MASC $^{28}$ ).

\section{Neurocognitive battery and composite scores}

A general-purpose neurocognitive test battery was selected. Owing to the unavailability of current, culturally appropriate, unbiased South African tests, ${ }^{29}{ }^{30}$ age-appropriate international tests with established utility in cross-cultural and multilingual contexts, and in SUD studies, were selected. In consultation with an Afrikaans linguistics specialist, appropriate cultural and language adaptations were made to the tests. Examples of these adaptations included replacement of items/terminology unfamiliar to South Africans, simplifications of test instructions, and substitution of items to ensure equivalent difficulty levels in Afrikaans and English. Test instructions, stimuli and response booklets were translated into Afrikaans and back-translated into English by independent translators. Nine composite domain scores were derived from the individual neurocognitive tests as follows:

1. Attention: Children's Color Trails Test $\left(\right.$ CCTT $\left.^{31}\right)$ times for trails 1 and 2; Children's Memory Scale
$\left(\mathrm{CMS}^{32}\right)$ numbers forward and backward subtests; Auditory Verbal Learning Test $\left(\mathrm{AVLT}^{33}\right)$ trial 1 score.

2. Coordination: Dominant and non-dominant peg insertion time, peg removal time and number of drops on the Grooved Pegboard Test $\left(\mathrm{GPT}^{34}\right)$.

3. Verbal reasoning: Vocabulary and Similarities subtests from the Wechsler Abbreviated Scale of Intelligence (WASI).

4. Non-verbal reasoning: Block Design and Matrix Reasoning subtests from the WASI ${ }^{35}$.

5. Verbal memory: AVLT immediate and delayed recall scores, and long-term per cent retention.

6. Visual memory: Rey-Osterrieth Complex Figure $\left(\mathrm{ROCF}^{36}\right)$ immediate and delayed recall scores; scores on trials 1-3, immediate and delayed recall of the CMS Dots subtest.

7. Verbal generativity: Phonemic fluency total score for letters L, B and S; semantic fluency total score for animal category.

8. Planning: ROCF copy; Tower of London, ${ }^{37}$ total correct score and total moves score.

9. Self-monitoring: error total on the CCTT (ie, nearmisses, prompts, colour sequence errors and number sequence errors); error total on phonemic and semantic fluency tasks (ie, rule violations, set-loss errors and repetitions); error total on the Tower of London (ie, rule and time violations).

\section{Procedure}

Participants were recruited from local schools of similar sociodemographic profiles within a 10 mile radius of the testing site at Tygerberg teaching hospital. Recruiting social workers gave educational talks regarding substance abuse in the classrooms; children were then invited to speak face-to-face and one-on-one with social workers. This first contact was used to screen for basic eligibility criteria, following which interested participants were provided with study information and consent forms to take home. Thereafter, recruiting social workers made telephonic or face-to-face contact with the children's parents/guardians, finalising written informed consent from parents/guardians and written assent from participants. Assenting participants were then invited to our testing site for the research visit, starting with a semistructured clinical evaluation conducted by a qualified psychiatrist. Participants also underwent physical and neurological examinations, venous blood sampling and urine drug screening. Of the participants at initial recruitment, exclusions were made on the basis of comorbid other drug use $(n=3)$, current severe mood disorder $(n=1)$ and repeatedly failing urine drug screens on the days of proposed testing $(n=1)$, resulting in a final sample of 40 participants.

Participants visited the testing site on two separate occasions to complete neurocognitive testing, at the baseline and at the follow-up visit. All testing was administered by trained professionals, and completed in participants' home language (English or Afrikaans). As per 
the exclusion criteria, participants underwent urine drug screening on each testing occasion. ${ }^{38}$

\section{Statistical analysis}

\section{Neurocognitive composite scores}

Where appropriate, neurocognitive test measures were reverse scored so that higher scores reflected better functioning. Thereafter, composite neurocognitive scores were computed to reduce the number of variables initially examined. A hybrid method (described by Medina et $a l^{39}$ ) grouped measures into domains based on theoretical assumptions, ${ }^{40}$ then average domain z-scores were computed. Goodness-of-fit was assessed by calculating Cronbach's $\alpha$ coefficients for each domain. We derived 10 composite neurocognitive domains, with Cronbach's $\alpha$ ranging from 0.59 to 0.86 . The tests in each composite are listed above, and in table 4 along with descriptive statistics for each measure.

After appropriate cross-checking and cleaning of data, statistical analysis was performed using SPSS V.17.0. Kolmogorov-Smirnov and Levene's tests were conducted to test for normality of data and homogeneity of variance, respectively. We examined group differences in demographic variables, as well as differences on outcome measures (within the same group over time, as well as across groups) using a series of analysis of variances. Although we explored a range of outcome measures in this analysis, the analysis was primarily of an explorative nature and so the threshold for statistical significance was set at 0.05 .

\section{RESULTS}

\section{Sociodemographic characteristics}

The groups were well-matched on key sociodemographic variables. While there were no significant between-group differences in age, years of successfully completed education, number of school grades repeated, or socioeconomic status (measured using a proxy measure of annual income) at either initial recruitment or follow-up, women and Afrikaans-speakers outnumbered men and English speakers, respectively (see table 1).

\section{Substance use}

Substance use data for both test occasions (baseline testing, and 12-month follow-up) appear in table 2. The participant groups were well differentiated with regard to substance use. With regard to baseline, and as expected, the Meth-cann group demonstrated significantly greater cannabis lifetime exposure than the Meth-only $(p<0.001)$ as well as the control $(p<0.001)$ groups, whose lifetime cannabis exposure was very low. Participants in the Meth-only and Meth-cann groups had consumed a significantly greater amount of methamphetamine $(\mathrm{p}=0.025$ and $\mathrm{p}=0.002$, respectively $)$ and nicotine ( $\mathrm{p}=0.005$ and $\mathrm{p}=0.014$, respectively) than participants in the control group; however, there was no difference in methamphetamine or nicotine exposure

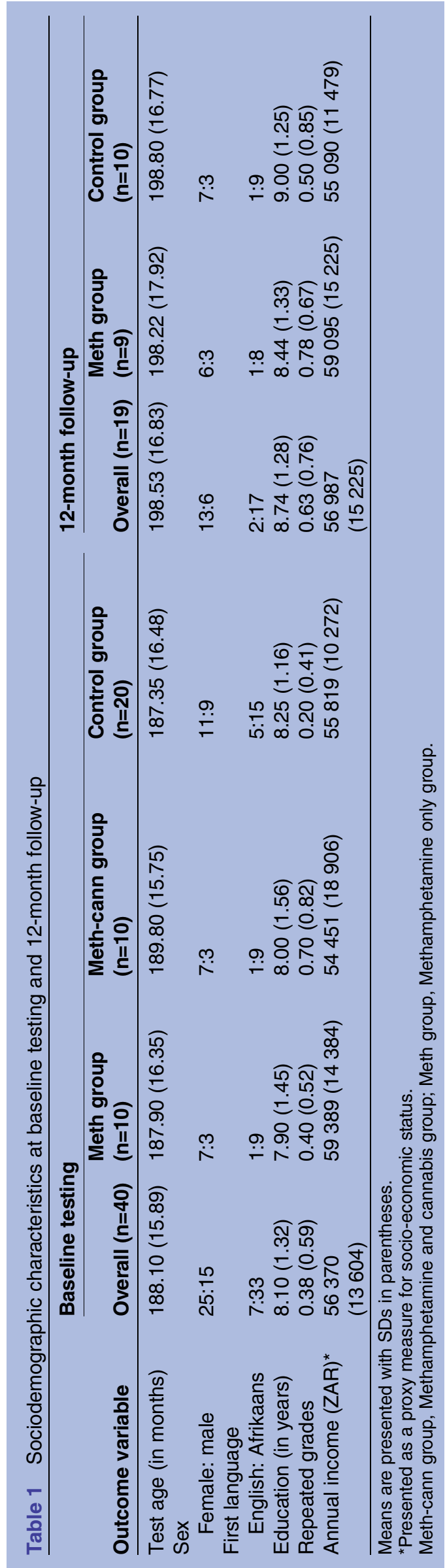


Table 2 Substance lifetime exposure across groups at baseline testing and 12-month follow-up

\begin{tabular}{|c|c|c|c|c|c|}
\hline \multirow[b]{2}{*}{ Outcome variable } & \multicolumn{3}{|l|}{ Baseline testing } & \multicolumn{2}{|l|}{ 12-month follow-up } \\
\hline & $\begin{array}{l}\text { Meth group } \\
(n=10)\end{array}$ & $\begin{array}{l}\text { Meth-cann group } \\
(n=10)\end{array}$ & $\begin{array}{l}\text { Control group } \\
(n=20)\end{array}$ & $\begin{array}{l}\text { Meth group } \\
(n=9)\end{array}$ & $\begin{array}{l}\text { Control group } \\
(n=10)\end{array}$ \\
\hline Alcohol (standard units) & $19.00(26.45)$ & $23.60(28.45)$ & 7.55 (13.84) & $51.00(58.30)$ & 22.90 (30.95) \\
\hline Nicotine (cigarettes) & $5088.10(7250.28)$ & 4431.40 (4012.89) & 286.90 (922.97) & $4843.49(3714.54)$ & $638.60(1443.24)$ \\
\hline Cannabis ('joints') & $5.70(5.40)$ & $917.70(854.19)$ & $0.10(0.31)$ & $151.67(331.94)$ & $0.20(0.42)$ \\
\hline $\begin{array}{l}\text { Methamphetamine } \\
\text { ('hits' smoked) }\end{array}$ & $534.20(531.20)$ & $736.80(514.53)$ & $0(0)$ & $527.56(522.82)$ & $0(0)$ \\
\hline
\end{tabular}

Means are presented with SDs in parentheses.

Meth-cann group, Methamphetamine and cannabis group; Meth group, Methamphetamine only group.

between the Meth-only and Meth-cann groups $(\mathrm{p}=0.546$ and $\mathrm{p}=0.725)$. The three groups did not differ with regard to alcohol exposure $(\mathrm{F}(2,37)=2.158, \mathrm{p}=0.130$, $\left.\eta^{2}=0.104\right)$.

At follow-up, there was no difference between the control and Meth-only groups with regard to alcohol or cannabis lifetime exposure $(\mathrm{p}=0.020$ and $\mathrm{p}=0.14$, respectively). Similar to baseline, participants in the Meth-only group had consumed more nicotine and methamphetamine than those in the control group at follow-up ( $p=0.004$ and $p<0.001$, respectively).

With regard to substance use trajectory, neither alcohol nor nicotine exposure changed significantly over time for either the control $(\mathrm{p}=0.069$ and $\mathrm{p}=0.423$, respectively) or Meth-only ( $\mathrm{p}=0.135$ and $\mathrm{p}=0.929$, respectively) group. Although cannabis use increased numerically in the Meth-only group over the test-retest interval, it was not significantly increased $(p=0.159)$. Cannabis exposure was also unchanged for the control group between baseline and follow-up $(\mathrm{p}=0.465)$. Methamphetamine exposure remained zero at both testing occasions for the control group, and did not increase significantly in the Meth-only group over the same period $(\mathrm{F}(17,19)=0.001, \mathrm{p}=0.978$, $\left.\eta^{2}=0.000\right)$. The absence of an increase in methamphetamine use in the Meth-only group over this period was due to the fact that most (6 of 9) participants had abstained from methamphetamine use for at least 6 months (ie, no longer endorsed current methamphetamine use disorders) between the two testing occasions.

\section{Psychopathology}

No participants endorsed diagnostically significant levels of psychopathology (aside from the relevant SUDs) at initial recruitment. Looking at subdiagnostic threshold symptom counts, the Meth-only group demonstrated significantly greater externalising symptoms $(\mathrm{CD}$ and oppositional disorder symptom counts) than the control group at baseline $(\mathrm{p}=0.024)$ and at follow-up $(\mathrm{p}=0.043)$. In contrast, externalising symptom counts were not elevated in the Meth-cann group at baseline. There were no other significant between-group differences in non-substance-related symptom counts. There were also no significant differences between baseline and follow-up in either the Meth-only or control groups. These clinical data are presented in table 3 .

\section{Neurocognitive performance}

At baseline, there were significant group differences in neurocognitive test performance in the non-verbal reasoning, verbal memory, planning and self-monitoring

Table 3 Subdiagnostic psychopathology and self-reported anxiety across groups at baseline testing and 12-month follow-up

\begin{tabular}{|c|c|c|c|c|c|}
\hline \multirow[b]{2}{*}{ Outcome variable } & \multicolumn{3}{|c|}{ Baseline testing } & \multicolumn{2}{|c|}{ 12-month follow-up } \\
\hline & $\begin{array}{l}\text { Meth group } \\
(\mathrm{n}=10)\end{array}$ & $\begin{array}{l}\text { Meth-cann group } \\
(\mathrm{n}=10)\end{array}$ & $\begin{array}{l}\text { Control group } \\
(n=20)\end{array}$ & $\begin{array}{l}\text { Meth group } \\
(\mathrm{n}=9)\end{array}$ & $\begin{array}{l}\text { Control group } \\
(n=10)\end{array}$ \\
\hline \multicolumn{6}{|c|}{ Sub-diagnostic symptom counts } \\
\hline ADHD & $3.50(6.19)$ & $4.30(5.33)$ & $0.80(1.64)$ & $3.56(7.07)$ & 3.60 (8.33) \\
\hline PTSD & $2.40(4.53)$ & $1.70(1.83)$ & $2.35(4.39)$ & $1.67(4.27)$ & $1.50(2.76)$ \\
\hline $\begin{array}{l}\text { Externalising } \\
\text { composite }^{*}\end{array}$ & $4.80(6.41)$ & $3.30(4.08)$ & $0.60(1.70)$ & $7.44(5.70)$ & $1.90(5.32)$ \\
\hline \multicolumn{6}{|l|}{ MASC subtotals } \\
\hline Physical symptoms & $12.60(5.87)$ & $14.50(7.28)$ & $11.73(7.89)$ & $10.44(10.25)$ & $10.20(6.83)$ \\
\hline Harm avoidance & $17.50(5.38)$ & $18.70(3.71)$ & $17.40(5.10)$ & $19.44(4.98)$ & $19.50(3.27)$ \\
\hline Social anxiety & $12.60(4.55)$ & $14.00(5.83)$ & $12.00(7.51)$ & $8.11(6.83)$ & $9.40(2.22)$ \\
\hline Separation/panic & $9.30(5.23)$ & $10.90(5.90)$ & $8.80(5.57)$ & $9.00(7.23)$ & $8.60(4.03)$ \\
\hline Total score & $50.80(13.55)$ & $57.00(19.77)$ & $48.80(21.20)$ & $46.11(24.64)$ & $46.40(9.11)$ \\
\hline
\end{tabular}




\begin{tabular}{|c|c|c|c|c|c|}
\hline & \multicolumn{3}{|l|}{ Baseline testing } & \multicolumn{2}{|l|}{ 12-month follow-up } \\
\hline & Meth group $(n=10)$ & Meth-cann group $(n=10)$ & Control group $(n=20)$ & Meth group (n=9) & Control group $(n=10)$ \\
\hline Attention $(\alpha=0.74)$ & $-0.25(0.63)$ & $-0.43(0.61)$ & $0.19(0.75)$ & $0.02(0.22)$ & $0.25(0.20)$ \\
\hline \multicolumn{6}{|l|}{ CCTT } \\
\hline Trial 1 time & $25.50(10.63)$ & $25.10(10.38)$ & $19.55(6.59)$ & $19.11(6.92)$ & $18.90(7.53)$ \\
\hline Trial 2 time & $48.40(13.12)$ & $53.10(16.46)$ & $43.65(12.21)$ & $42.44(8.97)$ & $40.60(13.25)$ \\
\hline \multicolumn{6}{|l|}{ CMS } \\
\hline Numbers forward & $6.90(1.52)$ & $8.00(1.05)$ & $7.85(1.57)$ & $7.11(1.54)$ & $7.90(1.52)$ \\
\hline Numbers backward & $3.60(0.70)$ & $3.10(0.99)$ & $4.50(1.54)$ & $3.56(1.59)$ & $4.30(0.82)$ \\
\hline AVLT Trial 1 & $7.40(1.96)$ & $6.00(1.41)$ & $6.95(1.96)$ & $8.00(2.24)$ & $7.10(1.66)$ \\
\hline WASI Vocabulary subtest & $25.20(10.30)$ & $26.60(6.85)$ & $33.15(8.45)$ & $29.33(12.34)$ & $29.50(4.88)$ \\
\hline WASI Similarities subtest & $18.20(6.46)$ & $19.60(6.87)$ & $22.60(7.18)$ & $22.11(8.43)$ & $22.30(4.64)$ \\
\hline Non-verbal reasoning $(\alpha=0.78)$ & $-0.26(0.92)$ & $-0.47(0.61)$ & $0.34(0.80)$ & $0.05(0.30)$ & $0.07(0.25)$ \\
\hline WASI Block Design subtest & $22.80(10.29)$ & $22.40(10.76)$ & $31.00(11.74)$ & $28.00(14.93)$ & $26.30(12.13)$ \\
\hline WASI Matrix Reasoning subtest & $15.80(6.63)$ & $13.40(4.38)$ & $19.05(5.05)$ & $17.67(8.00)$ & $16.80(6.78)$ \\
\hline Verbal memory $(\alpha=0.86)$ & $0.20(0.99)$ & $-0.60(0.88)$ & $0.08(0.52)$ & $-0.25(0.26)$ & $0.49(0.24)$ \\
\hline \multicolumn{6}{|l|}{ AVLT } \\
\hline Immediate recall & $10.00(1.33)$ & $8.30(2.58)$ & $10.45(1.93)$ & $9.11(2.57)$ & $10.90(1.85)$ \\
\hline Delayed recall & $9.80(2.04)$ & $7.90(3.04)$ & $10.30(1.90)$ & $9.44(3.50)$ & $11.50(1.96)$ \\
\hline Long-term per cent retention & $94.91(31.34)$ & $76.61(18.12)$ & $87.19(11.11)$ & $84.01(24.48)$ & $100.95(23.70)$ \\
\hline Visual memory $(\alpha=0.79)$ & $-0.14(0.72)$ & $-0.13(0.62)$ & $-0.02(0.81)$ & $0.29(0.25)$ & $0.09(0.23)$ \\
\hline \multicolumn{6}{|l|}{ ROCF } \\
\hline Immediate recall & $19.25(6.51)$ & $18.00(8.01)$ & $17.28(7.29)$ & $19.33(6.51)$ & $19.60(4.20)$ \\
\hline Delayed recall & $18.45(5.90)$ & 17.55 (7.58) & $17.68(7.73)$ & $19.22(6.18)$ & $19.00(4.73)$ \\
\hline \multicolumn{6}{|l|}{ CMS Dots } \\
\hline Trials 1-3 & $17.30(3.13)$ & $16.90(1.97)$ & $18.10(3.82)$ & $20.22(3.63)$ & $18.50(3.57)$ \\
\hline Immediate recall & $5.50(1.78)$ & $6.00(1.63)$ & $6.10(1.68)$ & $6.33(1.94)$ & $6.00(2.06)$ \\
\hline Delayed recall & $5.40(1.58)$ & $5.80(1.69)$ & $6.20(1.64)$ & $6.56(1.88)$ & $5.80(1.99)$ \\
\hline Verbal generativity $(\alpha=0.59)$ & $-0.38(1.04)$ & $-0.06(0.57)$ & $0.23(0.72)$ & $0.17(0.29)$ & $0.03(0.25)$ \\
\hline Phonemic fluency: total words & $24.40(9.68)$ & $29.00(4.76)$ & $28.55(6.58)$ & $29.56(12.47)$ & $24.10(10.73)$ \\
\hline Semantic fluency: total words & $13.30(4.24)$ & $13.70(2.58)$ & $15.75(3.04)$ & $14.56(3.05)$ & $15.10(3.11)$ \\
\hline Planning $(\alpha=0.73)$ & $-0.13(0.66)$ & $-0.47(0.76)$ & $0.22(0.66)$ & $0.05(0.21)$ & $0.13(0.20)$ \\
\hline ROCF copy & $30.40(4.19)$ & $29.15(5.19)$ & $31.48(2.77)$ & $30.17(4.57)$ & $30.55(3.02)$ \\
\hline \multicolumn{6}{|l|}{ Tower of London } \\
\hline Total correct score & $3.40(1.51)$ & $2.80(1.40)$ & $3.30(1.38)$ & $3.89(1.45)$ & $3.50(1.35)$ \\
\hline Total moves score & $33.90(16.29)$ & $39.50(13.82)$ & $26.65(12.36)$ & $29.00(11.74)$ & $25.20(8.27)$ \\
\hline Self-monitoring & $-0.71(0.84)$ & $-0.56(0.99)$ & $0.63(0.40)$ & $-0.72(0.23)$ & $0.68(0.21)$ \\
\hline CCTT total errors & $0.60(0.97)$ & $1.10(0.99)$ & $0.65(0.81)$ & $0.89(1.05)$ & $0.80(1.03)$ \\
\hline Phonemic fluency errors & $3.60(4.86)$ & $1.60(0.97)$ & $0.80(1.06)$ & $2.78(3.49)$ & $0.90(1.10)$ \\
\hline Semantic fluency errors & $0.40(0.70)$ & $0.10(0.32)$ & $0.25(0.55)$ & $0.33(0.50)$ & $0.20(0.42)$ \\
\hline Tower of London total errors & $2.20(3.10)$ & $3.20(3.16)$ & $0.50(1.10)$ & $2.22(5.89)$ & $0.30(0.48)$ \\
\hline
\end{tabular}

Means are presented with SEs in parentheses.

AVLT, Auditory Verbal Learning Test; CCTT, Children's Color Trails Test Meth-cann group; CMS, Children's Memory Scale; Methamphetamine and cannabis group; Meth group,

Methamphetamine only group; ROCH, Rey-Osterrieth Complex Figure; WASI, Wechsler Abbreviated Scale of Intelligence. 
domains. For each of these domains, the Meth-cann group had impaired performance relative to the control group (non-verbal reasoning: $\mathrm{p}=0.012$; verbal memory: $\mathrm{p}=0.024$, planning: $\mathrm{p}=0.013$ and self-monitoring: $\mathrm{p}<0.001)$. Additional group differences at baseline were observed in the verbal memory domain where the Meth-cann group performed worse than the Meth-only group ( $\mathrm{p}=0.023)$; and in the self-monitoring domain where the Meth-only group performed worse than the control group $(p<0.001)$. Impaired self-monitoring in the Meth-only group relative to the control group was maintained at follow-up $(p=0.003)$. There were no other significant differences either between groups at baseline or follow-up, or within groups between baseline and follow-up. Descriptive statistics for individual neurocognitive tests as well as neurocognitive composite domains appear in table 4 .

\section{DISCUSSION}

The main finding of this study was twofold. First, combined abuse of methamphetamine and cannabis was associated with relatively more widespread neurocognitive impairment than methamphetamine abuse alone. Second, increased externalising psychopathology and poorer self-monitoring associated with methamphetamine abuse appear to persist into abstinence.

Adolescents who abused methamphetamine alone showed some limited neurocognitive dysfunction in the self-monitoring domain; however, adolescents who abused cannabis as well as methamphetamine were susceptible to additional and more widespread cognitive dysfunction across several domains including verbal memory, planning and non-verbal reasoning. Although cannabis is currently the most common secondary illicit substance for methamphetamine users, ${ }^{19}$ few studies have examined the outcomes of concurrent methamphetamine and cannabis consumption. Our finding appears to stand in contrast to earlier preclinical work suggesting that methamphetamine neurotoxicity in fact attenuates the impact of certain cannabis-related behavioural and cognitive sequelae in rats. ${ }^{41}$ Similarly, an earlier clinical study showed no significant differences on neurocognitive outcomes in 'pure' methamphetamine abusers compared to those abusing methamphetamine and cannabis. ${ }^{42}$ Future research should aim to scrutinise the mechanisms of apparent differential effects of single versus concomitant drug use in order to clarify under which circumstances polysubstance use may provide a protective neurocognitive effect. ${ }^{21} 43$

The incidental finding that the Meth-only group largely abstained from methamphetamine use between baseline and follow-up assessments, teamed with overall consistency in the profiles of relative individual substance consumption between the control and Meth-only groups from baseline to follow-up (ie, the group differences in methamphetamine and nicotine exposure at baseline were maintained at follow-up, but not increased) provides an interesting perspective on the differential effects of current versus abstinent drug use. Since neither the control nor the Meth-only groups showed significant increases in lifetime exposure to any individual substance between baseline and follow-up, it seems probable that the clinical group differences that remained relevant from baseline to follow-up (increased externalising psychopathology and poorer self-monitoring) represent the enduring correlates of substance exposure established at baseline. Indeed, this proposition is supported by other studies showing that elevated subdiagnostic externalising psychopathology as well as deficits in executive functions such as self-monitoring may persistent long after substance use has ceased. ${ }^{23} 44$

A potential limitation of this study is that follow-up assessments were limited to Meth-only and control participants, and therefore comment on the long-term neurocognitive effects of concomitant methamphetamine and cannabis abuse is beyond the scope of this paper. A strength and potential limitation of this study is that we focus on the correlates of substance abuse during adolescence, a critical phase of neuromaturation. It must be noted that, although the sample size was small, the findings are valuable for application within this important developmental group. However, the applicability of these findings to adult drug-using populations with longer drug use histories remains to be seen. Furthermore, relatively longer-term follow-up studies of similar adolescent samples would be useful in order to examine the longevity of substance-related effects on neurocognition.

In summary, our findings demonstrate (1) the differential consequences on adolescent brain function and behaviour of methamphetamine abuse with or without cannabis, and (2) the differential acute and long-term effects of methamphetamine abuse in adolescence. These observations contribute to accumulating literature describing clinically relevant correlates of adolescent substance abuse. Given these findings, future studies should aim to clarify the specificity and longevity of clinical and neurocognitive effects of 'pure' methamphetamine abuse versus concurrent methamphetamine and cannabis use by examining current users with longer substance abuse histories, as well as abstinent users at relatively longer follow-up intervals.

\section{Author affiliations \\ ${ }^{1}$ Department of Psychiatry and Mental Health, University of Cape Town, Cape Town, Western Cape, South Africa \\ ${ }^{2}$ Department of Psychiatry, University of Stellenbosch, Cape Town, South Africa \\ ${ }^{3}$ Brain Institute, University of Utah School of Medicine, Salt Lake City, Utah, USA \\ ${ }^{4}$ Department of Psychiatry, University of Utah School of Medicine, Salt Lake City, Utah, USA}

Funding This work was supported by grant R21 DA021422-01 awarded by NIDA.

Competing interests None.

Patient consent Obtained. 
Ethics approval The Western Cape Education Department and Stellenbosch University's Ethics Committee granted written approval for the study, which was conducted in adherence to the guidelines contained in the Declaration of Helsinki.

Provenance and peer review Not commissioned; externally peer reviewed.

Data sharing statement No additional data are available.

Open Access This is an Open Access article distributed in accordance with the Creative Commons Attribution Non Commercial (CC BY-NC 4.0) license, which permits others to distribute, remix, adapt, build upon this work noncommercially, and license their derivative works on different terms, provided the original work is properly cited and the use is non-commercial. See: http:// creativecommons.org/licenses/by-nc/4.0/

\section{REFERENCES}

1. Chu P-W, Seferian KS, Birdsall E, et al. Differential regional effects of methamphetamine on dopamine transport. Eur J Pharmacol 2008;590:105-10.

2. Chang L, Alicata D, Ernst T, et al. Structural and metabolic brain changes in the striatum associated with methamphetamine abuse. Addiction 2007;102:16-32.

3. Eckstrand KL, Ding Z, Dodge NC, et al. Persistent dose-dependent changes in brain structure in young adults with low-to-moderate alcohol exposure in utero. Alcohol Clin Exp Res 2012;36:1892-902.

4. Schwendt M, Rocha A, See RE, et al. Extended methamphetamine self-administration in rats results in a selective reduction of dopamine transporter levels in the prefrontal cortex and dorsal striatum not accompanied by marked monoaminergic depletion. J Pharmacol Exp Ther 2009;331:555-62.

5. McCann UD, Kuwabara H, Kumar A, et al. Persistent cognitive and dopamine transporter deficits in abstinent methamphetamine users. Synapse 2008;62:91-100.

6. Nordahl TE, Salo R, Leamon M. Neuropsychological effects of chronic methamphetamine use on neurotransmitters and cognition: a review. J Neuropsychiatry Clin Neurosci 2003;15:317-25.

7. Vorhees CV, Skelton MR, Grace CE, et al. Effects of (+)-methamphetamine on path integration and spatial learning, but not locomotor activity or acoustic startle, align with the stress hyporesponsive period in rats. Int J Dev Neurosci 2009;27:289-98.

8. Derauf C, LaGasse LL, Smith LM, et al. Prenatal methamphetamine exposure and inhibitory control among young school-age children. $J$ Pediatr 2012;161:452-9.

9. Lu LH, Johnson A, O'Hare ED, et al. Effects of prenatal methamphetamine exposure on verbal memory revealed with fMRI. J Dev Behav Pediatr 2009;30:185-92.

10. Smith LM, LaGasse LL, Derauf $C$, et al. Motor and cognitive outcomes through three years of age in children exposed to prenatal methamphetamine. Neurotoxicol Teratol 2011;33:176-84.

11. Crane NA, Schuster RM, Fusar-Poli $P$, et al. Effects of cannabis on neurocognitive functioning: recent advances, neurodevelopmental influences, and sex differences. Neuropsychol Rev 2013;23:117-37.

12. Dean AC, Groman SM, Morales AM, et al. An evaluation of the evidence that methamphetamine abuse causes cognitive declines in humans. Neuropsychopharmacology 2013;38:259-74.

13. Lisdahl KM, Gilbart ER, Wright NE, et al. Dare to delay? The impacts of adolescent alcohol and marijuana use onset on cognition, brain structure, and function. Front Psychiatry 2013;4:53.

14. King G, Alicata D, Cloak C, et al. Neuropsychological deficits in adolescent methamphetamine abusers. Psychopharmacology (Berl) 2010;212:243-9.

15. Andersen SL. Stimulants and the developing brain. Trends Pharmacol Sci 2005;26:237-43.

16. Lubman DI, Yücel M, Hall WD. Substance use and the adolescent brain: a toxic combination? J Psychopharmacol 2007;21:792-4.

17. Scott JC, Woods SP, Matt GE, et al. Neurocognitive effects of methamphetamine: a critical review and meta-analysis. Neuropsychol Rev 2007;17:275-97.

18. Plüddemann A, Myers BJ, Parry $\mathrm{CDH}$. Surge in treatment admissions related to methamphetamine use in Cape Town, South Africa: implications for public health. Drug Alcohol Rev 2008;27:185-9.
19. Agrawal A, Neale MC, Prescott CA, et al. Cannabis and other illicit drugs: comorbid use and abuse/dependence in males and females. Behav Genet 2004;34:217-28.

20. Churchwell JC, Carey PD, Ferrett HL, et al. Abnormal striatal circuitry and intensified novelty seeking among adolescents who abuse methamphetamine and cannabis. Dev Neurosci 2012;34:310-17.

21. Couwenbergh $\mathrm{C}$, van den Brink W, Zwart K, et al. Comorbid psychopathology in adolescents and young adults treated for substance use disorders: a review. Eur Child Adolesc Psychiatry 2006;15:319-28.

22. Kaufman J, Birmaher B, Brent D, et al. The Schedule for Affective Disorders and Schizophrenia for School Aged Children (6-18 years) Lifetime Version. Retrieved 25 March 2010, from University of Pittsburgh, Department of Psychiatry. http://www.wpic.pitt.edulksads

23. American Psychiatric Association. DSM-IV-TR: diagnostic and statistical manual of mental disorders. Washington DC: American Psychiatric Publishing, 2000.

24. Fein G, Di Sclafani V, Finn P, et al. Sub-diagnostic psychiatric comorbidity in alcoholics. Drug Alcohol Depend 2007;87:139-45.

25. Di Sclafani V, Finn P, Fein G. Treatment-naive active alcoholics have greater psychiatric comorbidity than normal controls but less than treated abstinent alcoholics. Drug Alcohol Depend 2008;98:115-22.

26. Clark DB, Cornelius JR, Kirisci L, et al. Childhood risk categories for adolescent substance involvement: a general liability typology. Drug Alcohol Depend 2005;77:13-21.

27. American Psychiatric Association. DSM-5: diagnostic and statistical manual of mental disorders. Washington DC: American Psychiatric Publishing, 2013.

28. March JS. Multidimensional anxiety scale for children: technical manual. Toronto, ON: Multi-Health Systems, 1997.

29. Foxcroft $\mathrm{C}$, Paterson $\mathrm{H}$, le Roux $\mathrm{N}$, et al. Psychological assessment in South Africa: a needs analysis. The test use patterns and needs of psychological assessment practitioners. Pretoria: Human Sciences Research Council, 2004.

30. van Ommen $\mathrm{CH}$. Putting the $\mathrm{PC}$ in IQ: images in the Wechsler Adult Intelligence Scale-third edition (WAIS-III). S Afr J Psychol 2005;35:532-54.

31. Llorente AM, Williams J, Satz P, et al. Children's color trails test: professional manual. Odessa: Psychological Assessment Resources, 2003.

32. Cohen M. Chuldren's memory scale: manual. San Antonio, TX The Psychological Corporation, 1997.

33. Maj M, D'Elia L, Satz P, et al. Evaluation of two new neuropsychological tests designed to minimize cultural bias in the assessment of HIV-1 seropositive persons: a WHO study. Arch Clin Neuropsychol 1993;8:123-35.

34. Russell EW, Starkey RI. Halstead Russell Neuropsychological Evaluation System (HRNES). Los Angeles, CA: Western Psychological Services, 1993.

35. Wechsler D. Wechsler Abbreviated Scale of Intelligence. San Antonio, TX: The Psychological Corp, 1999.

36. Meyers J, Meyers K. Rey Complex figure and the recognition trial: professional manual. Supplemental norms for children and adolescents. Odessa: Psychological Assessment Resources, 1996.

37. Culbertson WC, Zillmer EA. Tower of London: technical manual. New York, NY: Multi-Health Systems Inc, 2001.

38. World Medical Association. Declaration of Helsinki 52nd WMA General Assembly. Edinburgh, Scotland, 2000.

39. Medina KL, Hanson KL, Schweinsburg AD, et al. Neuropsychological functioning in adolescent marijuana users: subtle deficits detectable after a month of abstinence. J Int Neuropsychol Soc 2007;13:807-20.

40. Lezak M, Howieson D, Loring D. Neuropsychological assessment New York, NY: Oxford University Press, 2004

41. Bortolato M, Frau R, Bini V, et al. Methamphetamine neurotoxicity increases brain expression and alters behavioral functions of CB1 cannabinoid receptors. J Psychiatr Res 2010;44:944-55.

42. Gonzalez R, Rippeth JD, Carey CL, et al. Neurocognitive performance of methamphetamine users discordante for history of marijuana exposure. Drug Alcohol Depend 2004;76:181-90.

43. Sung $\mathrm{YH}$, Carey PD, Stein DJ, et al. Decreased frontal $\mathrm{N}$-acetylaspartate levels in adolescents concurrently using both methamphetamine and marijuana. Behav Brain Res 2013;1246:154-61.

44. Volkow ND, Chang L, Wang GJ, et al. Loss of dopamine transporters in methamphetamine abusers recovers with protracted abstinence. J Neurosci 2001;21:9414-18. 